University of Nebraska - Lincoln

DigitalCommons@University of Nebraska - Lincoln

2002

\title{
How does water freeze inside carbon nanotubes?
}

\author{
Kenichiro Koga \\ Cornell University, koga@cc.okayama-u.ac.jp \\ G.T. Gao \\ US Naval Academy, Annapolis, MD \\ Hideki Tanaka \\ OkayamaUniversity, htanakaa@cc.okayama-u.ac.jp \\ Xiao Cheng Zeng \\ University of Nebraska-Lincoln, xzeng1@unl.edu
}

Follow this and additional works at: https://digitalcommons.unl.edu/chemzeng

Part of the Chemistry Commons

Koga, Kenichiro; Gao, G.T.; Tanaka, Hideki; and Zeng, Xiao Cheng, "How does water freeze inside carbon nanotubes?" (2002). Xiao Cheng Zeng Publications. 95.

https://digitalcommons.unl.edu/chemzeng/95

This Article is brought to you for free and open access by the Published Research - Department of Chemistry at DigitalCommons@University of Nebraska - Lincoln. It has been accepted for inclusion in Xiao Cheng Zeng Publications by an authorized administrator of DigitalCommons@University of Nebraska - Lincoln. 


\title{
How does water freeze inside carbon nanotubes?
}

\author{
Kenichiro Koga ${ }^{\mathrm{a}, *}$, G.T. Gao ${ }^{\mathrm{b}}$, Hideki Tanaka ${ }^{\mathrm{c}}$, X.C. Zeng ${ }^{\mathrm{d}}$ \\ ${ }^{a}$ Department of Chemistry, Baker Laboratory, Cornell University, Ithaca, NY 14853-1301, USA \\ ${ }^{\mathrm{b}}$ Department of Chemistry, US Naval Academy, Annapolis, MD 21402, USA \\ ${ }^{\mathrm{c}}$ Department of Chemistry, Okayama University, 3-3-1, Tsushima, Okayama 700-8530, Japan \\ ${ }^{\mathrm{d}}$ Department of Chemistry and Center for Materials Research and Analysis, University of Nebraska, \\ Lincoln, NE 68588, USA
}

\begin{abstract}
Phase behavior of quasi-one-dimensional water confined inside a carbon nanotube is studied in the thermodynamic space of temperature, pressure, and diameter of the cylindrical container. Four kinds of solid-like ordered structures - ice nanotubes - form spontaneously from liquid-like disordered phases at low temperatures. In the model system that comprises of TIP4P water molecules interacting with each other via short-range Lennard-Jones and long-range Coulomb site-site potentials under a periodic boundary condition in the axial direction, the phase change occurs either discontinuously or continuously depending on the path in the thermodynamic space. That the isotherms for a given diameter are found to be similar to those around the liquid-gas critical point of fluids suggests existence of a phase boundary terminated by a critical point. The apparently-complex phase behavior is accounted for by noting that the phase boundaries are layered surfaces in the three-dimensional thermodynamic space and some of the surfaces are terminated by critical lines.

(c) 2002 Elsevier Science B.V. All rights reserved.
\end{abstract}

PACS: 61.46.+w; 64.70.Dv; 68.08.De

Keywords: Confined water; Carbon nanotube; Phase behavior

\section{Introduction}

Phase behavior of water is very rich; there are 13 polymorphic phases of bulk ice identified experimentally thus far [1], the high- and low-density amorphous phases observed experimentally [2,3], and the high- and low-density liquid phases suggested by

\footnotetext{
* Corresponding author. Permanent address: Department of Chemistry, Fukuoka University of Education, Munakata, Fukuoka 811-4192, Japan. Tel.: +1-607-255-3845; fax: +1-607-255-4137.

E-mail address: kk275@cornell.edu (K. Koga).
} 
simulations in the experimentally inaccessible region [4,5]. Recent studies of phase behavior of water confined in narrow spaces have suggested the possibility of various condensed phases of water, and phase transitions between them in quasi-two-dimensional (Q2D) water [6,7]. Here, we present some recent results on water confined in very narrow cylindrical spaces - quasi-one-dimensional (Q1D) water-under various thermodynamic conditions. Specifically, we examine water confined inside carbon nanotubes since they provide well-defined cylindrical spaces and can be wet by low-surfacetension liquids including water [8].

Encapsulation of a second phase inside carbon nanotubes offers a new avenue to investigate dimensionally confined phase transitions $[9,10]$. When pure liquid water is encapsulated inside narrow carbon nanotubes, water molecules would be expected to line up into some quasi-one-dimensional structures, and on freezing, may exhibit quite different crystalline structures from bulk ice. Crystallization of water is known to be highly non-universal [11], i.e., the stable (or metastable) ice phase selected upon crystallization depends sensitively on the external environment. Thus, the first question we try to address is what type of crystalline lattice could be selected on cooling the confined water in narrow carbon nanotubes. Confinement may change not only resulting crystalline structures but also the way liquids freeze. For example, it has been conjectured [12] that in carbon nanotubes confined matter may exhibit an uncommon solid-liquid critical point [13] beyond which distinction between solid and liquid no longer exists. Thus the second question to be raised here is how water freezes and melts inside carbon nanotubes.

\section{Model system}

The model system consists of $N$ molecules confined inside a cylindrical pore of diameter $D$. The intermolecular interaction is taken to be the TIP4P water whose potential energy is given by the sum of the long-range Coulomb potential and the short-range Lennard-Jones (LJ) potential between the interaction sites. The potential function is truncated at $8.75 \AA$ by a switching function. The force field of the model carbon nanotube is taken to be a LJ potential integrated over the cylindrical area of the tube. A periodic boundary condition is applied in the axial direction (the $z$-direction) of the cylindrical unit cell of length $l$. In the molecular dynamics simulation the temperature $T$ and the axial pressure $P_{z z}$ are controlled, thus the total energy and the length $l$ fluctuate. The 'zigzag' $(R, 0)$ carbon nanotube considered here is specified by the index $R$, which is the number of 'benzene' rings along the circumference and, thus, proportional to $D$. For example, $R=14$ corresponds to $D=11.1 \AA$ and $R=18$ to $D=14.2 \AA$. Other details for the MD simulations and free-energy calculations are given elsewhere $[14,15]$.

\section{Structures of Q1D water at low temperatures}

Shown in Fig. 1 are structures of the TIP4P water in the cylindrical hollow space of the model carbon nanotubes at low- and high-temperatures. The low- $T$ phases are those reached by cooling stepwise the high- $T$ phases under $P_{z z}=50 \mathrm{MPa}$. It is clear that the high- $T$ phases have disordered structures with incomplete hydrogen-bond networks 


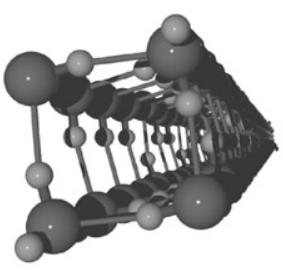

(a)

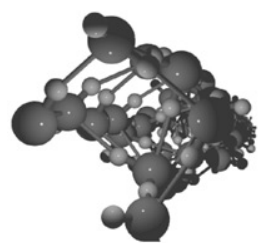

(e)

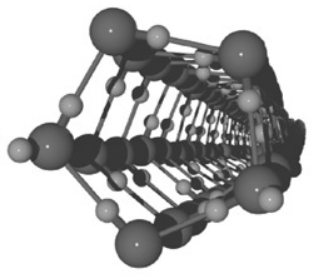

(b)

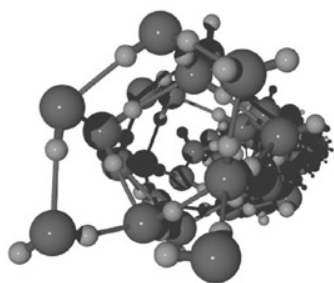

(f)

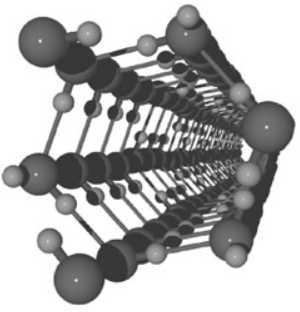

(c)

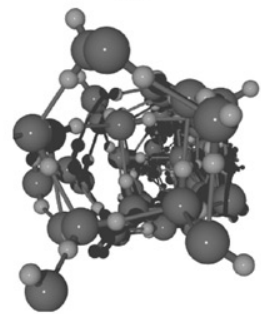

(g)

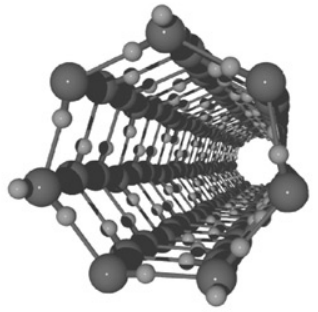

(d)

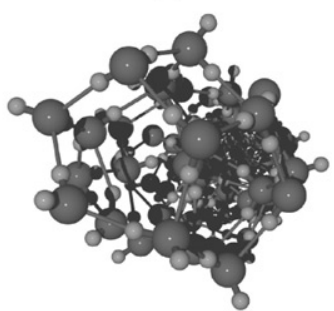

(h)

Fig. 1. (a) Square, (b) pentagonal, (c) hexagonal, (d) heptagonal ice nanotubes at $240 \mathrm{~K}$ (except d at $230 \mathrm{~K}$ ) in the carbon nanotubes of $R=14,15,16$, and 17 , and $(\mathrm{e})-(\mathrm{h})$ the corresponding liquid phases at $320 \mathrm{~K}$ (except $\mathrm{h}$ at $300 \mathrm{~K}$ ). See text for index $R$. The ice nanotubes form upon cooling under the axial pressure of $50 \mathrm{MPa}$ in MD simulations. Shown here are potential-energy local minimum structures.

whereas the low- $T$ phases have ordered structures with perfect networks. Specifically, the low- $T$ phases are Q1D $n$-gonal 'ice nanotubes' [16] composed of $n$-membered rings. The square $(n=4)$ ice nanotube forms in the $R=13$ and 14 systems, the pentagonal, hexagonal, and heptagonal ice nanotubes are found in the $R=15,16$ and 17 systems, respectively. All the Q1D ice nanotubes satisfy the ice rule as bulk ice does: every molecule is hydrogen-bonded to exactly four nearest neighbor molecules. From the ice rule it follows that the $\mathrm{OH}$ 'arms' of molecules along each edge of $n$-gonal prism are either all up or all down and that those along each $n$-membered ring line up either clockwise or counterclockwise. Another rule special for the Q1D ice is that the all-up and all-down $\mathrm{OH}$ arms are found alternatively. (In the cases of $n$ odd, this rule is not fully satisfied.) Besides their regular structures the low- $T$ phases have diffusivity as small as that of bulk ice [14], which indicates that the 'ice' nanotubes are indeed solids or solid-like.

\section{Phase behavior of $Q 1 D$ water}

Now we consider how the liquid-like disordered phases turn into the solid-like phases. Fig. 2 shows molar volume changes against temperature for six systems $(R=$ $13, \ldots, 18)$ under $50 \mathrm{MPa}$. For $R=16$ and 17 , there are discontinuous changes in volume and hysteresis loops. It is meant by 'discontinuous' that there is a sudden change of the quantity when measured in units of $5 \mathrm{~K}$. These discontinuous changes 

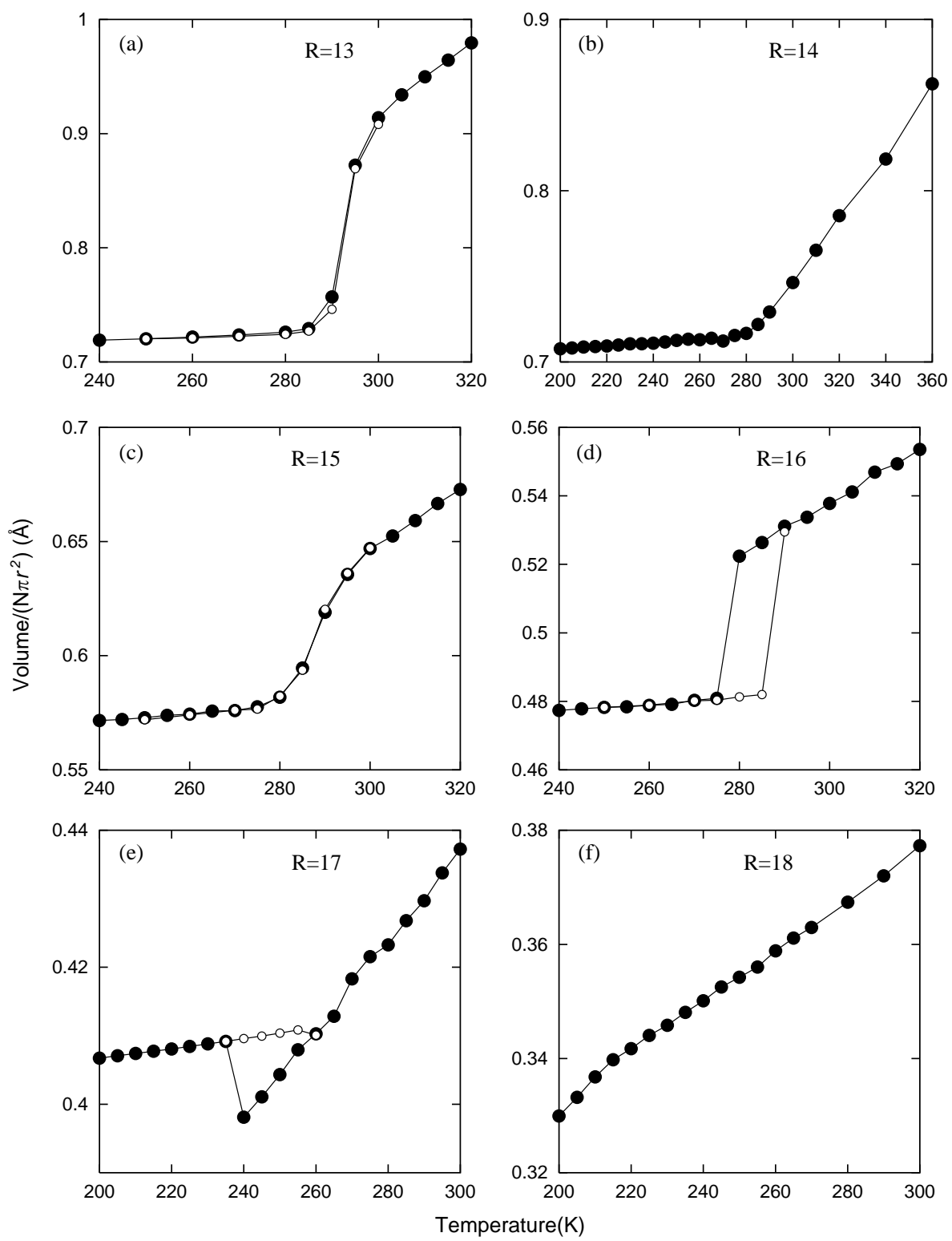

Fig. 2. (a-f) Volume per molecule vs. temperature for water confined in the carbon nanotubes of $R=13-18$. The applied axial pressure is $50 \mathrm{MPa}$. Filled and open marks indicate the cooling and heating process, respectively.

correspond to structural changes between liquid-like and solid-like phases (hexagonal and heptagonal ice nanotubes). For $R=13,14$, and 15, the volume changes without any abrupt drop or jump and no hysteresis appears - an indication that the liquid-like 


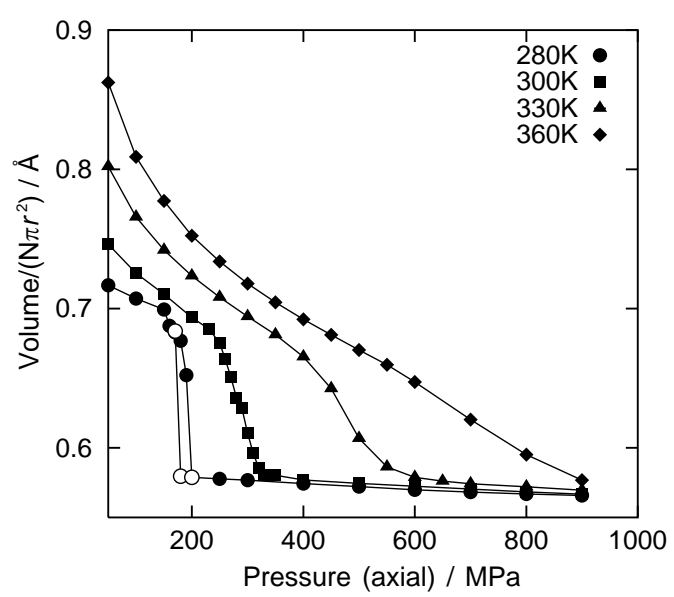

Fig. 3. Isotherms in the $P_{z z}-V$ plane for confined water in the $(14,14)$ carbon nanotube at temperature $280 \mathrm{~K}$ (circles), $300 \mathrm{~K}$ (squares), $330 \mathrm{~K}$ (triangles), and $360 \mathrm{~K}$ (diamonds). Filled and open marks indicate the compressing and decompressing process, respectively.

phase turns continuously into the solid-like phase (square for $R=13$ and 14 and pentagonal for $R=15$ ). In the $R=18$ system the liquid-like disordered phase remains at the lowest temperature. These results indicate that along the isobar $P_{z z}=50 \mathrm{MPa}$ confined water undergoes continuous phase changes to square $(n=4)$ and pentagonal $(n=5)$ ice nanotubes but discontinuous phase changes to hexagonal $(n=6)$ and heptagonal $(n=8)$ ice nanotubes. This does not mean, however, that the phase change is always continuous in the narrower carbon nanotubes; indeed richer phase behavior is found in the pressure-temperature plane at fixed $R$. For $R=15$ under $P_{z z}=500 \mathrm{MPa}$ the confined water exhibits on cooling a discontinuous phase change to the hexagonal ice nanotube, instead of the continuous change to pentagonal solid-like phase at $50 \mathrm{MPa}$. For $R=14$ under $P_{z z}=200 \mathrm{MPa}$ the water undergoes a discontinuous phase change to pentagonal ice nanotube, instead of the continuous change to the square solid-like phase at $50 \mathrm{MPa}$, and under $500 \mathrm{MPa}$ the liquid phase continuously turns into the pentagonal ice nanotube. These results illustrate complex phase behavior of water confined in the Q1D systems.

To better understand the phase behavior isotherms in the $P_{z z}-T$ plane were obtained at $R=14$ and 15. Fig. 3 shows the result for the $R=14$ system. At $280 \mathrm{~K}$, volume shows a sudden drop at $200 \mathrm{MPa}$ upon compressing and a sudden increase at $170 \mathrm{MPa}$ upon decompressing; i.e., a hysteresis loop is observed. Structural analysis shows that the low-density phase is a liquid-like disordered phase while the high-density phase is a solid-like pentagonal ice nanotube. The diffusion constant of the pentagonal nanotube (at $200 \mathrm{MPa}$ ) and the disordered phase (at $190 \mathrm{MPa}$ ) on compressing-path is $1.0 \times 10^{-10}$ and $9 \times 10^{-7} \mathrm{~cm}^{2} \mathrm{~s}^{-1}$, respectively. The diffusivity data reinforce that the high-density phase is solid-like while the low-density phase is liquid-like. At $300 \mathrm{~K}$, however, the transformation appears to be continuous since no abrupt jumps in volume are found. Long-time $(200 \mathrm{~ns})$ simulations were used in the pressure range between 250 and 
$330 \mathrm{MPa}$, in which the isotherm exhibits a marked decline. At $330 \mathrm{~K}$, the changes in volume become much less abrupt, and finally, at $350 \mathrm{~K}$, the changes in volume are essentially smooth. At the two higher temperatures, structural analysis shows that the high-density phase is a solid-like pentagonal-nanotube phase and the low-density phase is a liquid-like phase. In summary, the isotherms at $R=14$ seem analogous to those of fluids near the critical point. For the $R=15$ system, along the isotherms examined $(T=280,300,320 \mathrm{~K})$ the volume drops discontinuously at certain pressures on compressing. The low-density phases are liquid-like at these temperatures. (The pentagonal feature appears at $280 \mathrm{~K}$ though.) The high-density phases are the hexagonal ice nanotube (even at $320 \mathrm{~K}$ ). Unlike in the $R=14$ system, no continuous phase change from liquid-like to solid-like phases is found.

\section{Phase diagram}

Before we discuss the phase diagrams of confined water, some remarks are given on what we mean by 'phase boundary' in the Q1D systems. We note that although computer simulation cannot prove or disprove the existence of a true phase transition, or singularity in the partition function, in the thermodynamic limit $N \rightarrow \infty$, extrapolation of its results at finite $N$ to the thermodynamic limit is commonly accepted in 2D and 3D systems because we know there exist true phase transitions. A Q1D system, however, may or may not exhibit a true phase transition in the thermodynamic limit (depending on the range of the intermolecular interaction [17,18]), and we do not discuss here the existence of a true phase transition in the thermodynamic limit. On the other hand, our computer experiments demonstrate that water in carbon nanotubes exhibits abrupt changes in density, energy, entropy, and structure and corresponding hysteresis loops when certain paths are taken in the thermodynamic space. Furthermore, each single phase is well defined throughout the unit cell with length of the order of $10 \mathrm{~nm}$ (or $100 \AA$ ). It is in this sense that we use the terms 'phase transition' and 'phase boundary'; the concepts of phase boundary, phase transition, and phase diagram in Q1D systems are as useful as in $2 \mathrm{D}$ and $3 \mathrm{D}$ systems.

A schematic phase diagram for the $R=14$ system is given in Fig. 4. The phase boundary separates the high-density and low-density phases, which are referred to as pentagonal and square phases for convenience. At low temperatures (e.g., $200 \mathrm{~K}$ ) both the pentagonal and square phases are solid-like; at medium temperatures (e.g., $270 \mathrm{~K}$ ) the former is solid-like while the latter is liquid-like. Since the phase change becomes continuous above $280 \mathrm{~K}$, the phase boundary should terminate at some point around that temperature. The point is referred to as a 'critical point' in the same restricted sense discussed above. This phase diagram explains the complex phase behavior observed in the $R=14$ system. The continuous change from liquid-like structure to solid-like square ice nanotube at $50 \mathrm{MPa}$ on cooling (Fig. 2b) does not involve any crossing of the phase boundary; the path of $200 \mathrm{MPa}$ isobar crosses the phase boundary, thus the discontinuous change to the pentagonal phase; and the path of $500 \mathrm{MPa}$ isobar does not cross the boundary (thus the continuous change to the pentagonal phase) but does cross the extension of the phase boundary where a marked change is found. 


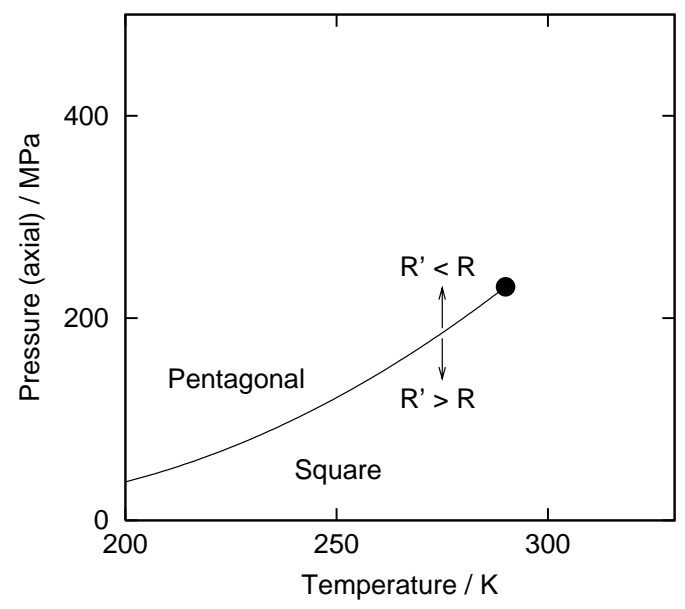

Fig. 4. A schematic $T-P_{z z}$ phase diagram of confined water in the $R=14$ system. The phase boundary (solid line) divides the low-density (square) and high-density (pentagonal) phases. At low temperatures both phases are solid-like, at medium temperatures the former is liquid-like while the latter is solid-like, and at the high temperatures both are liquid-like. The phase boundary terminates at a critical point around $280 \mathrm{~K}$. The arrows indicate the direction to which the phase boundary would be shifted if the diameter of the pore is slightly increased $\left(R^{\prime}>R\right)$ or decreased $\left(R^{\prime}<R\right)$.

For the $R=15$ system the low-density and high-density phases separated by the phase boundary are referred to as pentagonal and hexagonal phases because the former is a pentagonal ice nanotube at low temperatures while the latter is a solid-like hexagonal ice nanotube. Unlike in the $R=14$ system, no continuous change from one to the other phases is found in this system. Thus it seems that the phase boundary does not terminate at a critical point. For the $R=16$ systems, the low- $T$ and high-density phase is a solid-like hexagonal ice nanotube while the high- $T$ and low-density phase is liquid-like. No continuous path is found in this system. For the $R=17$ systems, the low- $T$ phase is a solid-like pentagonal ice nanotube and the high- $T$ phase is liquid-like. Note that the low- $T$ phase is a low-density phase unlike in the other systems (See Fig. 2). Thus the phase boundary should have a negative slope in the $T-P_{z z}$ plane.

Now we examine how the phase diagrams at discrete values of $R$ relate with each other. First we consider a system slightly wider than $R=14$. Then one would find the phase boundary shifted slightly downward with respect to that in the $R=14$ system. That is, the equilibrium pressure at given $T$ decreases with increasing the radius of the carbon nanotube. Le Chatelier's principle explains this qualitatively: increasing the radius has the effect of lowering the pressure $P_{N}$ exerted on the cylindrical wall, which in turn induces a process that increases $P_{N}$. The process is to drive the system to the pentagonal phase, because at the phase equilibrium $P_{N}$ exerted by the pentagonal portion is higher than that by the square portion. A more precise explanation is given by the Clapeyron equations for the quasi-one-dimensional system [15] by which one can obtain the rates of the boundary shift, $\left(\partial P_{z z}^{\mathrm{eq}} / \partial R\right)_{T}$ and $\left(\partial T^{\mathrm{eq}} / \partial R\right)_{P_{z z}}$. Indeed MD simulations at $R=14.2$ have shown the shift of the phase boundaries that is consistent with prediction from 
the Clapeyron equations. With increasing $R$ the square-pentagonal phase boundary with a critical point moves downward to low $P_{z z}$ and eventually disappears. At $R=15$ the square-pentagonal boundary no longer exists, and thus we observe the continuous change from a liquid-like state to a solid-like state of the pentagonal ice nanotube under $P_{z z}=50 \mathrm{MPa}$. However there is a pentagonal-hexagonal phase boundary at $R=15$. This boundary would moves upward to high- $P_{z z}$ with decreasing $R$ and is no longer found at $R=14$. At $R=13$, no discontinuity is found when the liquid-like state turns into the solid-like square ice nanotube. However, simulations at smaller $R$ show that there is a phase boundary that separates a low-density liquid-like phase and the solid-like square ice nanotube phase. Thus no discontinuity at $R=13$ is not because the system is too narrow to exhibit any phase transition but because it is too narrow for the square-pentagonal phase boundary and too wide for the liquid-square phase boundary.

In summary the global phase diagram in the Q1D system seems to be the following: the phase boundaries in the $T P_{z z} R$ space are found to be surfaces that have very steep slopes with respect to the $R$-axis, and two of them (the square-pentagonal and liquid-square phase boundary) appear to terminate at a 'critical line'.

\section{Acknowledgements}

KK wishes to thank Professor B. Widom for valuable discussions on the phase transition. KK and HT are supported by Japan Society for the Promotion of Science (JSPS), the Japan Ministry of Education, and IMS. XCZ is supported by US National Science Foundation and ONR. KK is visiting Cornell University under JSPS Fellowship for Research Abroad 2001.

\section{References}

[1] C. Lobban, J. Finney, W. Kuhs, J. Chem. Phys. 112 (2000) 7169.

[2] O. Mishima, L.D. Calvert, E. Whalley, Nature 310 (1984) 393.

[3] O. Mishima, L.D. Calvert, E. Whalley, Nature 314 (1984) 76.

[4] P.H. Poole, F. Sciortino, U. Essmann, H.E. Stanley, Nature 360 (1992) 324.

[5] H. Tanaka, Nature 380 (1996) 328.

[6] K. Koga, X.C. Zeng, H. Tanaka, Phys. Rev. Lett. 79 (1997) 5262.

[7] K. Koga, H. Tanaka, X.C. Zeng, Nature 408 (2000) 564.

[8] E. Dujardin, T.W. Ebbesen, H. Hiura, K. Tanigaki, Science 265 (1994) 1850.

[9] J. Sloan, J. Cook, M.L.H. Green, J.L. Hutchison, J. Mater. Chem. 7 (1997) 1087.

[10] X. Fan, E. Dickey, P. Eklund, K. Williams, L. Grigorian, R. Buczko, S. Pantelides, S. Pennycook, Phys. Rev. Lett. 84 (2000) 4621.

[11] D.W. Oxtoby, Nature 347 (1990) 725.

[12] P. Ball, Nature 361 (1993) 297.

[13] H.E. Stanley, Introduction to Phase Transitions and Critical Phenomena, Oxford University Press, New York, 1971.

[14] K. Koga, G.T. Gao, H. Tanaka, X.C. Zeng, Nature 412 (2001) 802.

[15] K. Koga, G.T. Gao, H. Tanaka, X.C. Zeng, in preparation.

[16] K. Koga, R.D. Parra, H. Tanaka, X.C. Zeng, J. Chem. Phys. 113 (2000) 5037.

[17] K. Johansson, J. Stat. Phys. 169 (1995) 521.

[18] J.L. Lebowitz, Rev. Mod. Phys. 71 (1999) S346. 